\title{
Searches for ultra long-lived particles with MATHUSLA
}

\author{
Emma Torró Pastor ${ }^{*}$ \\ University of Washington - Seattle \\ E-mail: Emma.Torro.Pastor@cern.ch
}

\begin{abstract}
With the current experiments at the particle accelerators, no search strategy will be able to observe the decay of neutral long-lived particles with masses above $\mathrm{GeV}$ and lifetimes at the limit set by Big Bang Nucleosynthesis, $c \tau \sim 107-108 \mathrm{~m}$. The MATHUSLA detector concept (MAssive Timing Hodoscope for Ultra-Stable neutraL pArticles) will be presented. It can be implemented on the surface above ATLAS or CMS detectors in time for the high-luminosity LHC operations, to search for neutral long-lived particles with lifetimes up to the BBN limit. The large area of the detector allows MATHUSLA to make important contributions also to cosmic-ray physics. We will also report on the analysis of data collected by the test stand installed on the surface above the ATLAS detector, the on-going background studies, and plans for the MATHUSLA detector. The observation of neutral long-lived particles at the LHC would reveal physics beyond the Standard Model and could account for the many open issues in our understanding of our universe. Longlived particle signatures are well motivated and can appear in many theoretical constructs that address the Hierarchy Problem, Dark Matter, Neutrino Masses and the Baryon Asymmetry of the Universe.
\end{abstract}

European Physical Society Conference on High Energy Physics - EPS-HEP2019 -

10-17 July, 2019

Ghent, Belgium

\footnotetext{
${ }^{*}$ Speaker.

${ }^{\dagger}$ On behalf of the MATHUSLA Collaboration
} 


\section{Introduction}

Long-lived particles (LLPs) occur in many extensions to the Standard Model (SM) with lifetimes that can be as long as the Big Bang Nucleosynthesis (BBN) bound of about $c \tau \lesssim 10^{7}-$ $10^{8} \mathrm{~m}$ [1]. Examples of models where such particles are predicted which can be produced at the Large Hadron Collider (LHC) include: Supersymmetric (SUSY) models such as RPV SUSY [2] and Stealth SUSY [3], models addressing the hierarchy problem such as Hidden Valleys [4], and models addressing dark matter [5].

The main experiments at the LHC have extensive programs to search for such particles, covering lifetimes from a few centimeters to tens of meters [6]. However, their reach is limited by different factors such as the trigger, the presence of backgrounds from the collision or from beam effects and ultimately by the size of the detectors. These limitations could risk missing a discovery should Ultra-Long-Lived Particles (ULLPs) be created at the LHC collisions.

MATHUSLA (MAssive Timing Hodoscope for Ultra-Stable neutraL pArticles) [7] is a proposed large-scale surface detector to be located above ATLAS [8] or CMS [9] to address a significant gap in the LHC's experiments coverage. The $\sim 90 \mathrm{~m}$ of rock between the interaction point (IP) and the detector's decay volume on the surface gives enough shielding for MATHUSLA to work in a clean environment. Being a background-free experiment increases the sensitivity to LLP lifetimes up to lifetimes of $10^{7} \mathrm{~m}$ and extends the sensitivity of the main detectors by orders of magnitude. LLP decays would be reconstructed as displaced vertices (DVs) of upwards traveling charged particles. As a secondary physics objective, MATHUSLA would also be able to perform cosmic ray physics measurements and help solve important puzzles in astroparticle physics.

A white paper describing the need for a detector like MATHUSLA was published by a large number of experimentalists and theorist in 2018 [10]. The MATHUSLA collaboration has made significant progress on detailed background and design studies, and presented a Letter of Intent [11] to the LHCC. A test stand with a detector layout similar to the one envisioned for the MATHUSLA detector was assembled in the surface above ATLAS and took data during 2018, analysis of which is ongoing. The results will provide empirical information on potential backgrounds coming from the LHC as well as from cosmic rays. The collaboration is now seeking to construct a MATHUSLA demonstrator detector unit by 2021. The full-scale detector could become operational by 2025-26.

\section{The MATHUSLA detector}

\subsection{Basic Detector Principles}

ULLPs near the BBN lifetime bound arising from exotic Higgs decays could be discovered if the detector had a linear size of $\sim 20 \mathrm{~m}$ in the direction of travel with a good geometric coverage ( $\sim 5 \%$ of solid angle), which results in a detector with linear dimensions of $\mathscr{O}(100 \mathrm{~m})$. The $\sim 90$ meters of rock between the collision point and the surface eliminates most backgrounds associated with $p p$ collisions. Still, a large background of cosmic muons and backgrounds from high energy muons and neutrinos coming from the IP must be rejected which requires good tracking and vertexing capabilities. The proposed detector, illustrated in the left panel of Figure 1 along with two possible DVs from LLP decays, is a large box of $100 \times 100 \times 25 \mathrm{~m}^{3}$ volume, with a robust tracking system on its upper part, $25 \mathrm{~m}$ air decay volume and a tracking veto on the floor. 

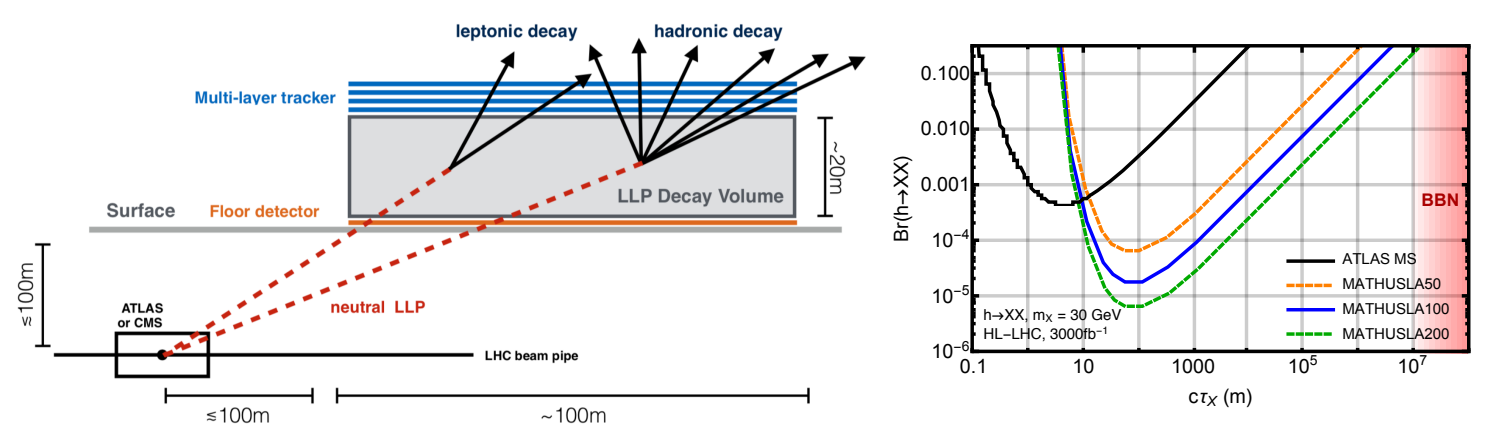

Figure 1: Left:. Simplified MATHUSLA layout. Right: Comparison of reach for $30 \mathrm{GeV}$ LLPs decaying hadronically, for various MATHUSLA geometries (curves correspond to 4 LLPs decaying in the detector volume) and the ATLAS exclusion projection using a single-DV search in the Muon System [12].

Three simplified geometries for the MATHUSLA decay volume were studied in the Letter of Intent. MATHUSLA200 has a $200 \mathrm{~m} \times 200 \mathrm{~m} \times 20 \mathrm{~m}$ decay volume. This large size would allow it to probe LLP lifetimes close to the BBN limit $c \tau \lesssim 10^{7} \mathrm{~m}$. MATHUSLA100 is smaller by a factor of 4 with a $100 \mathrm{~m} \times 100 \mathrm{~m} \times 20 \mathrm{~m}$ decay volume. MATHUSLA50, a $50 \mathrm{~m} \times 50 \mathrm{~m} \times 20 \mathrm{~m}$ decay volume, was also considered for comparison. Sensitivity projections for these geometries in the expected luminosity for the HL-LHC are shown in the right panel of Figure 1. They are compared to the exclusion projection using a single-DV search in the ATLAS Muon System [12].

The dominant background comes from cosmic rays (CRs), with a rate in the $\mathrm{MHz}$ range. Their rejection depends on the robust ceiling tracking system, with spatial and temporal resolutions in $\mathrm{cm}$ and nanosecond range, respectively. If the tracking layers span a vertical distance of a few meters, full 4-dimensional track and displaced vertex reconstruction is possible, which significantly reduces the combinatorial backgrounds as tracks must intersect in both space and time to form a vertex. Both Resistive Plate Chambers (RPCs) and plastic scintillators are time-tested technologies that meet the needed specifications. Since CRs travel downwards and do not inherently form DVs, this signal requirement is expected to allow MATHUSLA to reach the near-zero-background regime.

The expected rate of muons from LHC collision is about $10 \mathrm{~Hz}$. They are upwards traveling muons that do not generally produce a DV and that can be vetoed by the floor tracker. Upward going atmospheric neutrinos are estimated to be of order 10 to 100 per year and most can be rejected using time of flight. In addition, they can be measured when there are no LHC beams. Neutrinos from LHC collisions are a subdominant background, estimated to be a few events during the entire HL-LHC data taking, and can be rejected with geometrical cuts and timing vetoes on non-relativistic charged tracks associated with the scattering event.

\subsection{The modular concept}

MATHUSLA is designed to be a large area detector, requiring to cover a wide surface with detector material. Building MATHUSLA as an array of independent modules makes it a flexible and scalable detector, easy to adapt to the available land and to the specific site conditions. It also allows for a staged integration with an incremental ramp-up. One of the advantages of MATHUSLA is that it is entirely parasitic: its construction and operation are not expected to interfere with the 
operation of ATLAS or CMS and its staged construction can happen as a completely independent plan from that of the LHC and the experiments upgrade work.

The current design considers individual modules with a volume of $9 \mathrm{~m} \times 9 \mathrm{~m} \times 25 \mathrm{~m}$ and a separation of $\sim 1$ m between modules as shown in Figure 2. Each module includes two tracking layers on the floor to act as a veto for charged particles from the IP, an air-filled decay volume of $25 \mathrm{~m}$ and 5 tracking layers on the ceiling for track and vertex reconstruction. The extension of the decay volume by a few meters above ground has been decided as well as the inclusion of two extra tracking layers near the upper part of the decay volume to improve tracking and vertexing resolutions. Studies conclude that a scintillator veto surrounding the entire volume is not needed.

The baseline trigger system is driven by units of $3 \times 3$ modules, a choice based on the largest possible inclination angle for the MATHUSLA200 case which would be a very safe option for a $100 \mathrm{~m} \times 100 \mathrm{~m}$ detector.
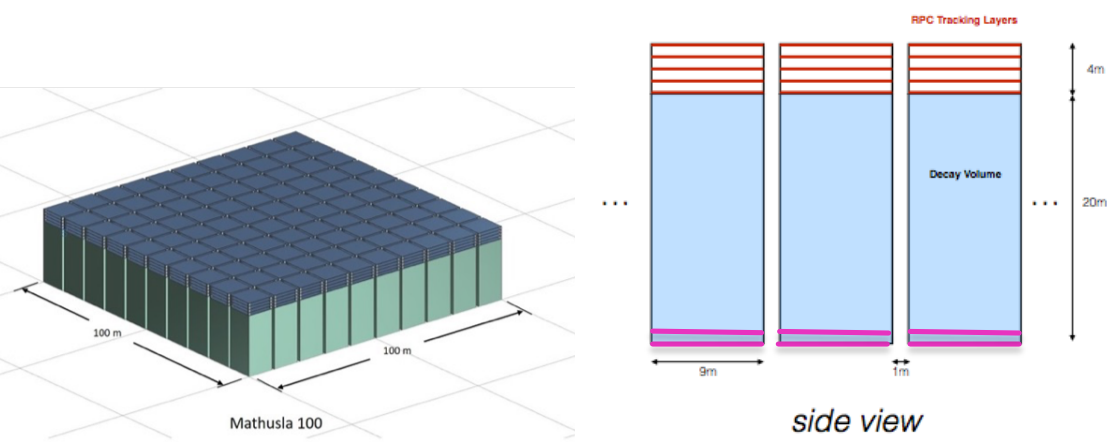

Figure 2: Schematics of the modular design of MATHUSLA (left) and structure of the modules (right).

\subsection{Current geometry proposal}

CERN owns an available piece of land near CMS that would be a suitable site for the detector [11]. The MATHUSLA collaboration is working with Civil Engineers from CERN to define the building and the layout of the detector. Figure 3 shows the details on the planned position (left) and size of the detector (right).
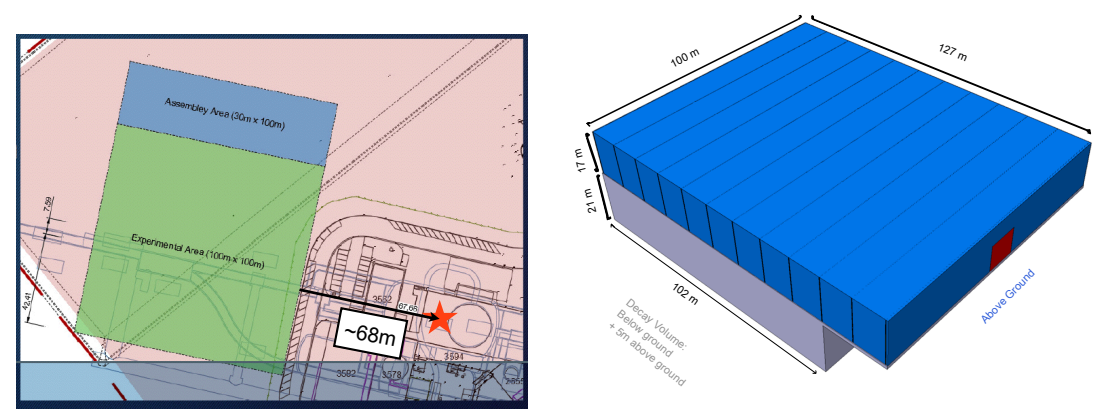

Figure 3: Left: CERN-owned land near CMS and location of MATHUSLA. The IP in CMS is marked as a red star. Right: Schematics showing the current proposed dimensions of the MATHSULA building. 
The current proposal contemplates a $100 \mathrm{~m} \times 102 \mathrm{~m}$ experimental area located on the surface of CMS together with a $30 \mathrm{~m} \times 100 \mathrm{~m}$ adjacent area for the detector assembly. A total height of $\sim 40 \mathrm{~m}$ includes a $\sim 25 \mathrm{~m}$ decay volume, $21 \mathrm{~m}$ of which would be excavated, and $12 \mathrm{~m}$ in the upper part to host the tracker and the cranes system for assembly and maintenance. Having a large part of the decay volume underground brings it closer to the IP, which increases the solid angle in the acceptance for LLPs generated in the collisions. To adjust to the available land, this proposal has a $7.5 \mathrm{~m}$ offset with respect to the center of the beams. The site allows for the detector to be as close as $68 \mathrm{~m}$ away from the IP.

The original MATHUSLA200 proposal assumed a distance of $100 \mathrm{~m}$ from the IP both horizontally and vertically. Reducing these distances as explained above, the current proposal can reach a similar LLP sensitivity as the MATHUSLA200 case with a final detector design more optimized, with smaller geometry that is costefficient and tailored to the available experimental site. Figure 4 shows the details on the proposed geometry. An enclosed building would contain an experimental area, an assembly area and a system of cranes. The $9 \mathrm{~m} \times$ $9 \mathrm{~m}$ modules would be arranged in three arrays, each of which would be served by an independent $20 \mathrm{~T}$ crane, reaching the assembly area for construction and maintenance.

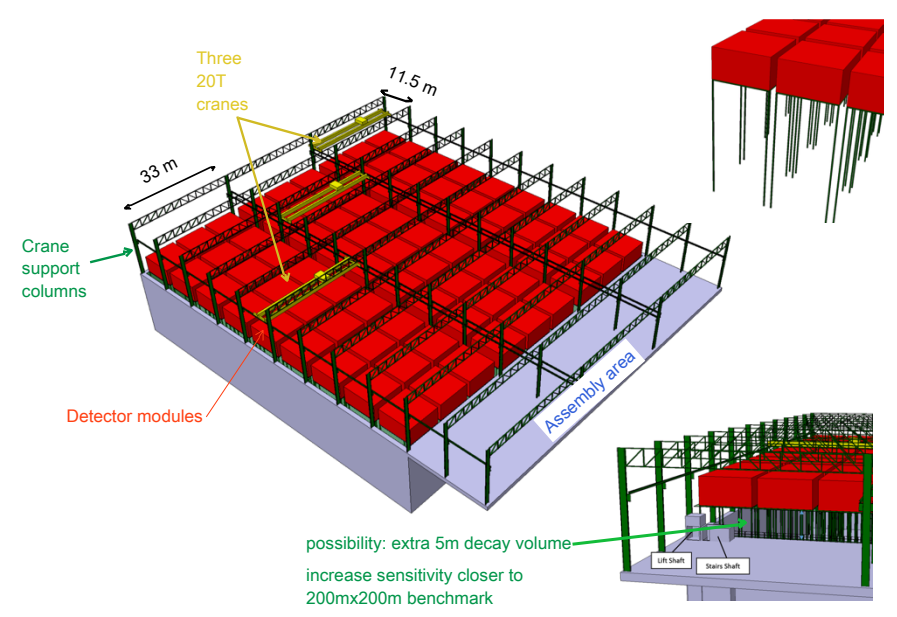

Figure 4: Current proposal for the modular design of MATHUSLA, the experimental and assembly areas and the cranes.

\section{The MATHUSLA test module}

The MATHUSLA detector has been conceived to be a background-free detector, an hypothesis that can be confirmed making use of a test stand detector. A test stand with a layout similar to the one envisioned for MATHUSLA was installed in the surface area above the ATLAS IP, taking data with different beam conditions during 2018. Data taken during periods with no beams present in the LHC provide a good measurement of the background from cosmic rays. Data collected with collisions at the LHC can give an estimate of the background expected from the LHC. A precise measure of the charged particle flux in the test stand will provide the veto efficiency requirement for the main detector to guarantee that no cosmic particles fake charged particles coming from the LHC. All this information can be used to optimize the design of the main detector.

Following the concept of the main detector, the test stand is composed of one external layer of scintillators in the upper part and one in the lower part with six layers of RPCs between them as shown in Figure 5. The overall structure is $\sim 6.5 \mathrm{~m}$ tall, with a base of $\sim 2.5 \times 2.5 \mathrm{~m}^{2}$. 
Events are selected by a trigger system that requires a hit in one of the scintillators layers followed by a hit in the opposite scintillator layer within a time window. Tracks are then reconstructed using a minimum $\chi^{2}$ fit using spatial and time information from the RPC and scintillator hits. Tracks are identified as upwards- or downwards going by the fit. Figure 6 shows the distribution of tracks as a function of the reconstructed zenit angle and a comparison to the expected distribution from a simulation. Data are separated in periods when no beam was present in the LHC (runs with no beam, blue line) and periods when there were collisions at the LHC (runs with beam, red line). In the case of downwards-going tracks (left), the angular distributions in runs with and without beam match perfectly. They are also compatible with the cosmic ray simulation dis-

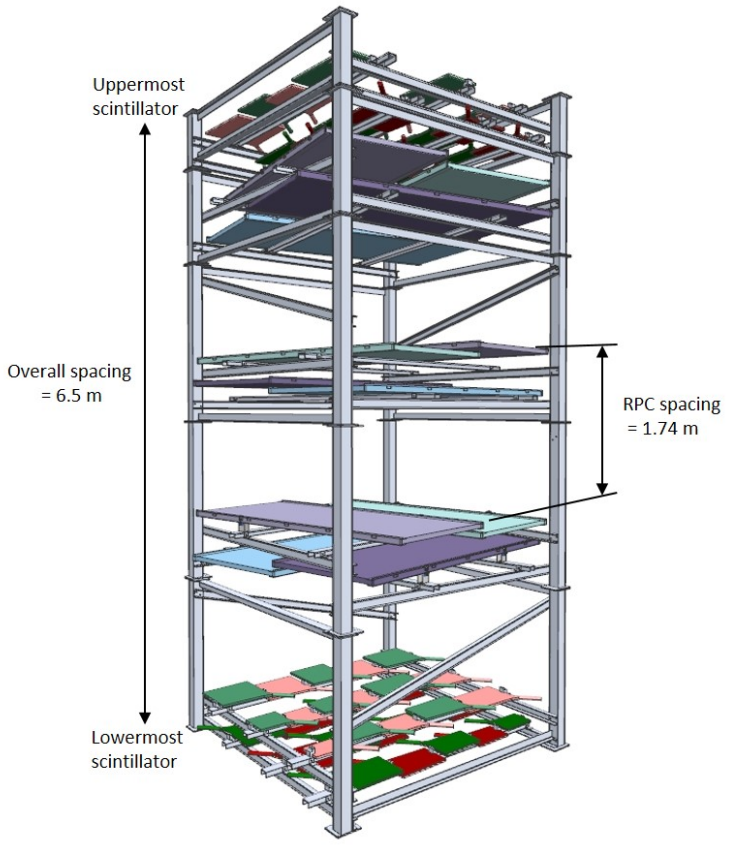

Figure 5: schematicviewoftheMATHUSLAteststand tribution (grey area) where multiple scattering of particles in the detector material has not been taken into account. This distribution confirms that the downwards-going tracks are properly reconstructed. In the case of upwards-going tracks (right), the distribution in events with no beam matches that of the downwards-going tracks. This conforms a background whose source is currently under study. In runs with beam, a peak is observed at small zenit angles, compatible with particles coming from the ATLAS IP, convoluted with the same background distribution observed in events with no beam. In this case the simulation shows the expected angular distribution from particles coming from the IP where multiple scattering of particles in the rock and in the detector material are not included.
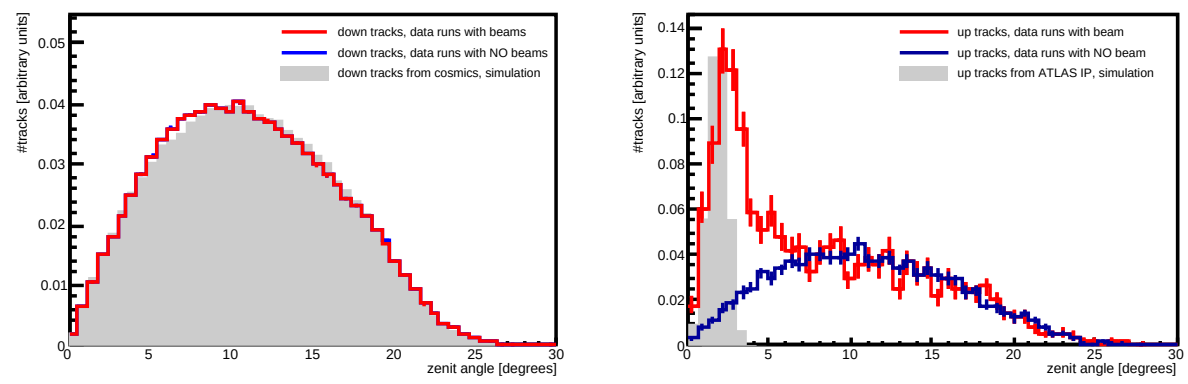

Figure 6: Distribution of reconstructed tracks as a function of the zenit angle. Data events in runs with (no) beam are shown as (blue) red lines. Left: downwards-going tracks, overlaid is a simulation of cosmics rays events. Right: upwards-going tracks, overlaid is a simulation of particles coming from the ATLAS IP. Multiple scattering has not been taking into account in the simulations. 


\section{MATHUSLA as a cosmic ray telescope}

MATHUSLA has all the qualities needed to act as an excellent cosmic ray (CR) telescope. MATHUSLA's large area gives it good efficiency for extended air showers from primary CRs. Its combination with high-resolution directional tracking and proximity to ATLAS or CMS for correlated shower core measurements could more detailed studies of the core structure. These measurements, which do not interfere with the primary goal of LLP discovery, represent a "guaranteed physics return" on the investment of the detector, as well as an opportunity to establish a cosmic ray physics program. The qualitative $\mathrm{CR}$ physics case was discussed in [10], with more detailed studies in progress.

\section{References}

[1] A. Fradette and M. Pospelov, BBN for the LHC: constraints on lifetimes of the Higgs portal scalars, Phys. Rev. D96 (2017), no. 7 075033, [arXiv:1706.01920].

[2] R. Barbier et al., R-parity violating supersymmetry, Phys. Rept. 420 (2005) 1-202, 10.1016/j.physrep.2005.08.006, [arXiv: hep-ph/ 0406039 ].

[3] J. Fan, M. Reece, J. T. Ruderman, Stealth Supersymmetry, JHEP 11 (2011) 12, 10.1007/JHEP11(2011)012, [arXiv:1105.5135 [hep-ph]].

[4] M. J. Strassler, K. M. Zurek, Echoes of a Hidden Valley at Hadron Colliders, Phys. Lett. B 651 (2007) 374-379, 10.1016/j.physletb.2007.06.055, [arXiv: hep-ph / 0604261 ].

[5] D. Tucker-Smith, N. Weiner, Inelastic dark matter, Phys. Rev. D 64 (2001) 043502, 10.1103/PhysRevD.64.043502, [arXiv: hep-ph/0101138].

[6] ATLAS Collaboration, Search for long-lived, weakly-interacting particles that decay to displaced hadronic jets in proton-proton collisions at $\sqrt{s}=8 \mathrm{TeV}$ with the ATLAS detector, Phys. Rev. D 92 (2015) 012010, 10.1103/PhysRevD.92.012010, [arXiv:1504.03634 [hep-ex]].

[7] J. P. Chou, D. Curtin, and H. J. Lubatti, New Detectors to Explore the Lifetime Frontier, Phys. Lett. B767 (2017) 29-36, [arXiv:1606.06298].

[8] ATLAS Collaboration, The ATLAS Experiment at the CERN Large Hadron Collider, 2008 JINST 3 S08003

[9] CMS Collaboration, CMS physics: Technical design report, CERN-LHCC-2006-001, CMS-TDR-008-1

[10] D. Curtin et al., Long-Lived Particles at the Energy Frontier: The MATHUSLA Physics Case, arXiv:1806.07396.

[11] C. Alpigiani et al., A Letter of Intent for MATHUSLA: a dedicated displaced vertex detector above ATLAS or CMS, CERN-LHCC-2018-025, LHCC-I-031 (2018) [arXiv: 1811.00927 ].

[12] A. Coccaro, D. Curtin, H. J. Lubatti, H. Russell, J. Shelton, Data-driven Model-independent Searches for Long-lived Particles at the LHC, Phys. Rev. D 94113003 (2016), [arXiv: 1605 . 02742] .

[13] D. Curtin and M. E. Peskin, Analysis of Long Lived Particle Decays with the MATHUSLA Detector, Phys. Rev. D97 (2018), no. 1 015006, [arXiv: 1705.06327 ]. 